\title{
Heiner Syndrome and Milk Hypersensitivity: An Updated Overview on the Current Evidence
}

\author{
Stefania Arasi $1, *,+, \ddagger \mathbb{D}$, Carla Mastrorilli ${ }^{2,+} \neq \pm \mathbb{D}$, Luca Pecoraro ${ }^{3,4, \ddagger}$, Mattia Giovannini ${ }^{5, \ddagger}$, Francesca Mori ${ }^{5, \ddagger}$, \\ Simona Barni ${ }^{5, \ddagger}$, Lucia Caminiti ${ }^{6, \ddagger}$, Riccardo Castagnoli ${ }^{7,} \mp \mathbb{D}$, Lucia Liotti ${ }^{8, \ddagger} \mathbb{D}$, Francesca Saretta ${ }^{9, \ddagger}$, \\ Gian Luigi Marseglia ${ }^{7, \ddagger}$ and Elio Novembre ${ }^{10, \ddagger}$
}

1 Translational Research in Pediatric Specialities Area, Division of Allergy, Bambino Gesù Children's Hospital, IRCCS, Piazza Sant'Onofrio, 4, 00165 Rome, Italy

2 Pediatric Unit and Emergency, University Hospital Consortium Corporation Polyclinic of Bari, Pediatric Hospital Giovanni XXIII, 70126 Bari, Italy; carla.mastrorilli@icloud.com

3 Department of Medicine, University of Verona, 37129 Verona, Italy; luca.pecoraro@assst-mantova.it

4 Pediatric Unit, ASST Mantua, 46100 Mantua, Italy

5 Allergy Unit, Department of Pediatrics, Meyer Children's University Hospital, 50139 Florence, Italy; mattia.giovannini@unifi.it (M.G.); francesca.mori@meyer.it (F.M.); simona.barni@meyer.it (S.B.)

6 Department of Human Pathology in Adult and Development Age "Gaetano Barresi", Allergy Unit, Department of Pediatrics, AOU Policlinico Gaetano Martino, 98158 Messina, Italy; lucycaminiti@yahoo.it

7 Department of Pediatrics, Pediatric Clinic, Fondazione IRCCS Policlinico San Matteo, University of Pavia, 27100 Pavia, Italy; riccardo.castagnoli@yahoo.it (R.C.); gl.marseglia@smatteo.pv.it (G.L.M.)

8 Department of Pediatrics, AOU Ospedali Riuniti Ancona, Presidio Ospedaliero di Alta Specializzazione "G. Salesi", 60126 Ancona, Italy; lucia.liotti@ospedaliriuniti.marche.it

9 Pediatric Department, Latisana-Palmanova Hospital, Azienda Sanitaria Universitaria Friuli Centrale, 33100 Udine, Italy; francescasaretta@gmail.com

check for

updates

Citation: Arasi, S.; Mastrorilli, C.;

Pecoraro, L.; Giovannini, M.; Mori, F.; Barni, S.; Caminiti, L.; Castagnoli, R.; Liotti, L.; Saretta, F.; et al. Heiner Syndrome and Milk Hypersensitivity: An Updated Overview on the Current Evidence. Nutrients 2021, 13, 1710. https://doi.org/10.3390/nu13051710

Academic Editor: Yvan Vandenplas

Received: 8 April 2021

Accepted: 17 May 2021

Published: 18 May 2021

Publisher's Note: MDPI stays neutral with regard to jurisdictional claims in published maps and institutional affiliations.

Copyright: (c) 2021 by the authors. Licensee MDPI, Basel, Switzerland. This article is an open access article distributed under the terms and conditions of the Creative Commons Attribution (CC BY) license (https:/ / creativecommons.org/licenses/by/ $4.0 /)$.
10 Department of Health Science, University of Florence, 70126 Florence, Italy; elio.novembre@meyer.it

* Correspondence: stefania.arasi@opbg.net; Tel.: +39-06-68593570

+ These authors contributed equally to this work.

$\ddagger$ From the Rare Allergic Diseases Commission of the Italian Society of Pediatric Allergy and Immunology.

Abstract: Infants affected by Heiner syndrome (HS) display chronic upper or lower respiratory tract infections, including otitis media or pneumonia. Clinically, gastrointestinal signs and symptoms, anemia, recurrent fever and failure to thrive can be also present. Chest X-rays can show patchy infiltrates miming pneumonia. Clinical manifestations usually disappear after a milk-free diet. The pathogenetic mechanism underlying HS remains unexplained, but the formation of immune complexes and the cell-mediated reaction have been proposed. Patients usually outgrow this hypersensitivity within a few years. The aim of this review is to provide an updated overview on the current evidence on HS in children, with a critical approach on the still undefined points of this interesting disease. Finally, we propose the first structured diagnostic approach for HS.

Keywords: allergy; anemia; cow's milk; children; immunology; non-IgE-mediated food allergy; pneumonia; pulmonary hemosiderosis; pulmonary infiltrates

\section{Introduction}

Heiner syndrome (HS) is a rare food-induced hypersensitivity disease characterized by chronic respiratory symptoms with X-ray (XR) infiltrates, and the resolution of signs and symptoms after the removal of milk proteins. Other clinical manifestations include poor growth, gastrointestinal signs and symptoms, iron deficiency anemia and pulmonary hemosiderosis $(\mathrm{PH})$. Precipitins to cow's milk (CM) were also considered a useful aid in recognizing hypersensitivity to $\mathrm{CM}$ [1]. The literature concerning HS is restricted to a few case reports or series, although the disease has always been described at infant or pediatric age [2]. The definition of the disease is lacking and the diagnosis is often delayed, since its presentation is uncommon with dissimilar manifestations. In the present review we 
aimed at presenting clinical, diagnostic and therapeutic characteristics of HS, starting from current evidence.

\section{Search Methodology and Results}

We carried out a non-systematic review including the most relevant studies on "Heiner Syndrome" (HS) present on databases including PubMed (https://www.ncbi.nlm.nih. gov / pubmed/ accessed on 26 March 2021), MEDLINE, The Cochrane Library, from their inception to 26 March 2021. The searched terms were "Heiner Syndrome" [all fields]; "pulmonary hemosiderosis" and "children" [all fields]; "pulmonary hemosiderosis" and "cow's milk" [all fields]; "pulmonary hemosiderosis" and "hypersensitivity" [all fields]. We found 16 studies. They were all clinical cases or consecutive case series, involving an overall pool of 61 patients. Findings were summarized narratively below for each study as well as in Table 1.

In order to better stratify the level of evidence for the diagnosis, we are herein proposing the first structured diagnostic criteria for HS to our best knowledge. This diagnostic approach consists of the following criteria:

(A) Pulmonary symptoms and XR infiltrates or pulmonary hemosiderosis (PH);

(B) Resolution after milk removal;

(C) Recurrence after milk reintroduction.

HS diagnosis $(\mathrm{HSD}):(\mathrm{A})+(\mathrm{B})=$ probable disease; $(\mathrm{A})+(\mathrm{B})+(\mathrm{C})=$ convincing disease (Figure 1).

PROBABLE

Heiner

Syndrome

$(A)+(B)$
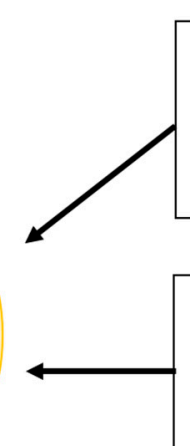

(B)

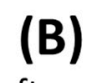

Resolution after milk removal

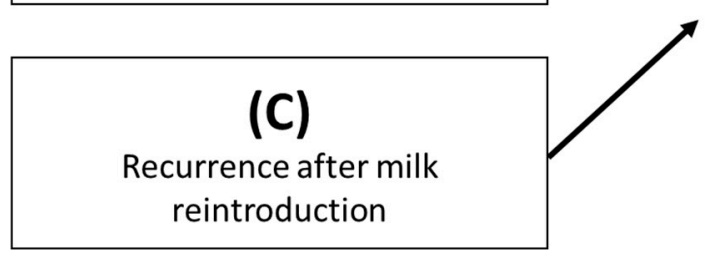

Figure 1. Proposed approach for Heiner syndrome diagnosis.

\section{CONVINCING}

Heiner

Syndrome

$(A)+(B)+(C)$ 
Table 1. Characteristics of the included studies of Heiner syndrome.

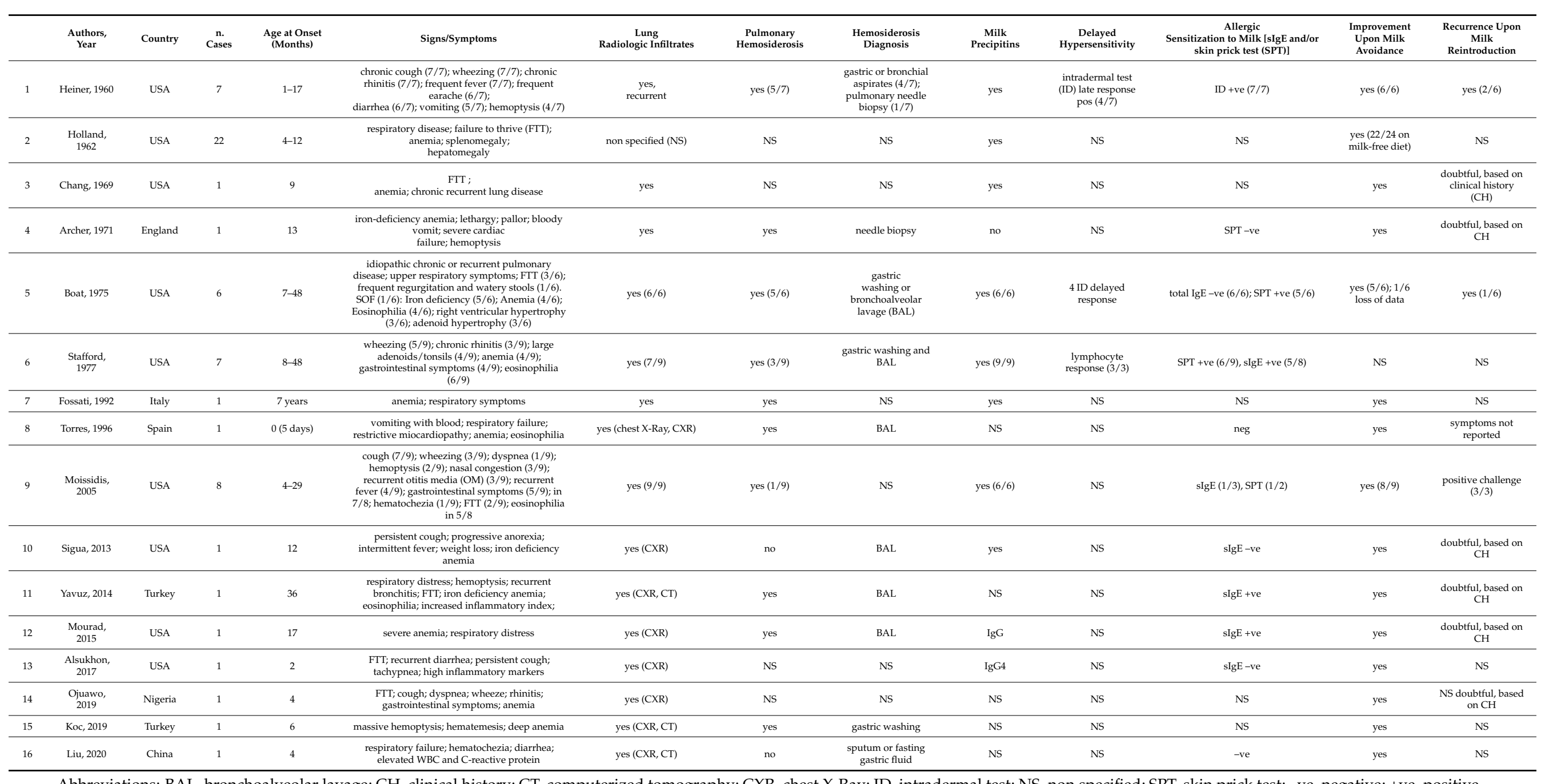

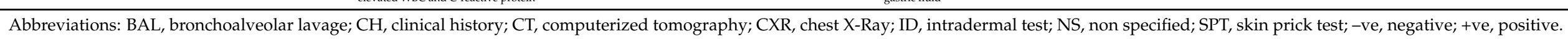


Milk precipitins have been evaluated only in some studies, mainly in the oldest ones. Based on current data and specifically due to the heterogeneity in the methodologies applied for laboratory tests, we decided to exclude laboratory parameters from the diagnostic approach.

(1) Heiner et al., 1960 [3]-In 1960, Heiner [3] (from who the name of the syndrome originates) first reported a chronic respiratory disease associated with multiple CM precipitins in the sera of seven children aged 6 weeks to 17 months. All the patients presented a chronic respiratory disease with maximal severity of clinical manifestations at the time of introduction of raw CM in the diet associated with the other signs and symptoms that define the syndrome, mostly iron deficiency anemia, gastrointestinal signs and symptoms, poor growth and $\mathrm{PH}$ documented by gastric or bronchial aspirates. Six out of seven patients overcame their disease by changing the milk content (i.e., milk processing or exclusion of milk proteins) in their diet, by using evaporated or boiled milk $(n=2)$, extensively hydrolyzed casein formula $(n=1)$ or soymilk $(n=3)$. Overall, the milk protein avoidance resulted in the complete disappearance of clinical manifestations and remission of the hematologic status. One patient spontaneously overcame the disease without dietary restriction between 2.5 to 3.5 years (y) of age. When milk was reintroduced after avoidance, two out of six showed a clinical and imaging relapse, and four became tolerant or partially tolerant to milk after intervals without clinical manifestations on a restricted diet ranging from three to six months in duration. Comment: The first description of HS. HSD: Convincing in two cases; probable in four patients.

(2) Holland et al., 1962 [4] - Stimulated by Heiner's observations, Holland et al. examined serum specimens from 1618 infants and children with the same technique, finding precipitins in 87 of them [4]. Patients of this population showed signs and symptoms suggestive of the syndrome but also different clinical manifestations, such as isolated upper respiratory diseases, hepatosplenomegaly and congenital heart diseases. Only 17 patients were reported to show respiratory signs and symptoms. No data on X-rays were reported. Because of the heterogeneity of the clinical manifestations and the limited number of diagnostic exams in this population, we selected 22/87 patients who improved during the CM diet period. No attempt of reintroduction was performed. Comment: We extrapolated 22 cases with suspected HS from a large and heterogeneous cohort. HSD: Nobody with probable or convincing clinical criteria.

(3) Chang et al., 1969 [5] - Chang described the clinical case of a 9-month-old girl admitted to the hospital for failure to thrive (FTT), anemia and chronic recurrent lung disease starting in the first weeks of age [5]. She underwent a lengthy diagnostic process, until the finding of milk precipitins suggested an HS diagnosis. The patient was then placed on a soymilk diet with clinical resolution. Flare-up signs and symptoms and radiological relapse due to the poor adherence to the diet are mentioned. However, controlled milk reintroduction was not performed. Comment: Single case report. No detailed data are reported about the follow-up. HSD: Probable.

(4) Archer, 1971 [6]-Archer reported the clinical case of a 13-month-old girl with a severe heart failure based on a profound iron deficiency anemia and idiopathic $\mathrm{PH}$, diagnosed by needle biopsy [6]. All of the immunological tests performed in order to investigate a CM sensitization, including serum precipitins, skin prick test (SPT) and immunoglobulin, were negative. Notwithstanding, a milk-free diet was commenced with a good clinical and radiological response. The first relapse during her first week with a childminder was probably due to the inadvertent administration of CM. Comment: The girl was admitted one year before with some symptoms and treated successfully with antibiotics. Results of CM reintroduction doubtful. HSD: Probable.

(5) Boat et al., 1975 [7]-In this study, 6 children with high titers of milk precipitins were identified by screening the sera of 160 children with idiopathic chronic lung disease associated with typical manifestations of milk-induced PH [7]. Elimination of CM 
from the diet, symptomatic therapy and adenoidectomy (when indicated) resulted in improvement in six out of six patients. Comment: Even if six out of six patients recovered in 5-21 days after milk removal, only one was rechallenged (Patient $C$ ) and one (Patient B) developed pneumonia within six months upon CM reintroduction. HSD: Convincing in one case; probable in five patients.

(6) Stafford et al., 1977 [8]—Nine patients with respiratory signs and symptoms and milk precipitins were enrolled in this study in order to elucidate the immunopathologic mechanisms involved in milk-induced $\mathrm{PH}$. No demonstration of a unique immunologic mechanism associated with milk-induced $\mathrm{PH}$ in the patients studied [8]. No clinical data about CM withdrawal were reported. Comment: Focus on immunological patho-mechanisms with poor clinical description of the enrolled participants. HSD: Nobody with probable or convincing criteria.

(7) Fossati, 1992 [9] - A 7-year-old girl was admitted to hospital because of anemia, and a PH was diagnosed [9]. Precipitating antibodies were also found. A marked improvement of clinical manifestations and XR results were found after removal of CM from the diet. Comment: Single clinical case report. No data on reintroduction. HSD: Probable.

(8) Torres et al., 1996 [10] - This study provides an interesting immunological overview on the HS based on data from a single clinical case of a girl [10]. Specifically, the authors speculate that an inflammatory response occurred after CM intake in the presented clinical case. A 5-day-old female newborn was admitted in an emergency department because of a severe bradycardia due to a myocarditis associated with assessed PH and anemia. Although the total specific IgE and specific IgG to milk proteins were below the detection limits, the patient underwent two oral food challenges $(\mathrm{OFC})$. During the in vivo tests, hematocrit, histamine, tryptase and ECP (eosinophil cationic protein) in blood and BAL, and N-methyl-histamine (NMH) in urine were measured before and at multiple times during the administration of standard formula (first OFC) and non-milk enteral nutrition (second OFC). When the girl was fed with CM, a remarkable increment of all the tested inflammatory mediators was reported; conversely, the hemoglobin level dropped significantly. Then, the same OFC was done with non-milk enteral nutrition with any variation being registered. These data are unfortunately not supported by clinical and radiologic information. Challenge. Comment: Unique case report of $\mathrm{PH}$ in which milk OFC induced an increase of inflammatory mediators suggesting a T cell-mediated pathogenesis of HS. HSD: Probable.

(9) Moissidis et al., 2005 [11]—Moissidis et al. reviewed eight cases of children affected by upper respiratory tract symptoms [11]. All cases presented radiological imaging with pulmonary infiltrates, and one had HP (defined as iron-laden macrophages in the bronchoalveolar lavage, gastric washing and open lung biopsy). Seven out of eight had gastrointestinal symptoms. High titers of precipitating antibodies to CM proteins were demonstrated in six out of six patients studied. However, HS was confirmed by the improvement of the clinical and radiological findings after a CM-free diet and relapse when a reintroduction was attempted in three out of six cases. Comment: The most detailed paper on the topic. However, cases were evaluated at different times and under different circumstances; therefore, specific data were not available for each patient. HSD: Convincing in three cases; probable in five patients.

(10) Sigua et al., 2013 [12] - A 12-month-old boy with multifocal pneumonia that was refractory to protracted antibiotic treatment was suspected to suffer from HS [12]. The clinical history showed that the boy underwent a milk-free diet from the first to the tenth month of age because of suspected non-IgE-mediated CM non-bloody diarrhea. $\mathrm{HS}$ appeared at $\mathrm{CM}$ reintroduction. Serum-precipitating IgG antibodies to all nine $\mathrm{CM}$ protein fractions tested were strongly positive. He underwent a strict soy-based diet from 12 months of age with prompt clinical remission and complete resolution of the previously identified pulmonary opacities at a chest X-ray performed at 14 months. 
Comment: A single patient with HS after a previous history of non-IgE-mediated CM gastrointestinal symptoms. HSD: Probable.

(11) Yavuz et al., 2014 [13] - A 3-year-old boy was referred to the emergency service with respiratory distress and hemoptysis [13]. Because of iron deficiency anemia, a BAL cytological examination was performed in order to confirm a PH. Precipitins were not determined. The patient overcame the disease through a CM avoidance diet. However, a low dose of both prednisolone and azathioprine was also prescribed. Furthermore, authors described that the patient in the next five years had many relapses because of failure to receive the prescribed medications and poor adherence to the diet. Moreover, during a hemoptysis attack, he showed new symptoms, such as edema, hematuria and hypertension. On this occasion, rapidly progressive glomerulonephritis was diagnosed on the basis of the histopathological findings and treated with a combination of cyclophosphamide and methylprednisolone. Comment: In this case report, an elimination diet and drugs were administrated together for an extended period of time, and during the follow-up the compliance was scarce. Therefore, it is difficult to differentiate the effects of each treatment and the actual cause-effect relationship. HSD: Probable.

(12) Mourad et al., 2015 [14] - The clinical case of a 17-month-old boy with idiopathic PH was described by Mourad et al. [14]. BAL demonstrated an abundance of fresh red blood cells and iron-laden macrophages. The CM-specific IgE level was only slightly elevated $(1.42 \mathrm{IU} / \mathrm{mL})$. IgG antibody levels to $\mathrm{CM}$ proteins were markedly elevated. In spite of the severity of the clinical conditions (i.e., severe anemia and respiratory failure with acidosis), the strict CM-free diet allowed the boy to overcome the disease. Hydrocortisone was also administrated, but it is not clear when it was introduced and for how long. A relapse was reported because the mother, while on raw $\mathrm{CM}$ avoidance, started feeding the patient with baked CM products. Comment: Single clinical case report. Hydrocortisone was also used. Controlled reintroduction not performed. HSD: Probable.

(13) Alsukhon et al., 2017 [15] —A 2-month-old male with recurrent diarrhea and FTT had persistent cough, tachypnea and high inflammatory markers despite antibiotic therapy for pneumonia [15]. An amino acid-based formula gave improvement in inflammation and respiratory function. Comment: Single case report. Milk reintroduction not performed. HSD: Probable.

(14) Ojuawo et al., 2019 [16] -Ojawo et al. described the clinical case of a 16-week-old boy with FTT, dyspnea and anemia who acceded to the emergency department in Nigeria [16]. Neither antibiotic treatment nor sodium citrate, administered for the suspicion of a renal tubular acidosis, modified his condition. Diagnosis of HS was based on the constellation of clinical features, XR results, and subsequent resolution after stopping CM. Parents on a follow-up visit reported occasional cough and rhinitis whenever CM was reintroduced. Comment: Single case report. No controlled CM reintroduction reported. HSD: Probable.

(15) Koc et al., 2019 [17] — A 6-month-old infant with massive hemoptysis, hematemesis and deep anemia was treated for bronchopneumonia four times [17]. When he was admitted to the emergency department, both chest- $X$ ray and computerized tomography documented many lung opacities and hemosiderin-laden macrophages were found in the patient's fasting stomach fluid examination, confirming the diagnosis of $\mathrm{PH}$. The boy was discharged with a CM-free diet, with complete clinical and radiological recovery. Comment: No laboratory data were reported, and no milk reintroduction test was reported. HSD: Probable.

(16) Liu et al., 2020 [18] — Liu et al. described a 4-month-old boy with a chronic pulmonary syndrome whose main presenting symptom was a persistent hematochezia since the tenth day of life [18]. Gastrointestinal endoscopic biopsy showed granulation tissue infiltrated by acute and chronic inflammatory cells, including some eosinophils. Additionally, in this case, the improvement of both clinical and radiologic findings 
after the elimination of milk suggested the diagnosis of HS. In addition to the CM elimination diet, the patient was treated with methylprednisolone $(1 \mathrm{mg} / \mathrm{kg})$ and montelukast. Comment: Single case report. No milk reintroduction test reported. HSD: Probable.

\section{Discussion}

To the best of our knowledge, the present article represents the first review on this rare disease. The data shown suggest a critical approach to the disorder (Table 1).

\subsection{Age at Onset}

The clinical onset of the disease has been described typically by the age of 1 month to 48 months, but it can develop even during the first 5 days of life, as reported by Torres et al. [10]. However, it can also appear later (the oldest patient was 5 years old) [4]. Nevertheless, there was a frequent delay in diagnosing this disease, due to its various modes of presentation and lack of standardized diagnostic criteria. The past medical history of affected children was always unremarkable. A family history of allergic disorders was often present. Differently from immediate-onset IgE-mediated CM allergy, HS did not display signs and symptoms before several days or weeks after $\mathrm{CM}$ consumption.

\subsection{Etiology}

Although HS is more likely to be induced by homogenized CM, the disease also may occur in some infants fed with CM-derived formula. Furthermore, it has been speculated that it can be related to non-IgE-mediated allergy to food proteins differently from $\mathrm{CM}$ at an older age (e.g., soy, egg, pork, wheat and peanut) [14,19]. In this context, a single case of $\mathrm{PH}$ hemosiderosis due to buckwheat has also been reported [20].

\subsection{Clinical Characteristics}

Respiratory features of the disease included persistent cough, dyspnea, tachypnea, wheezing, occasional sputum production and rales. The peculiarity of pneumonia in these case series was the refractoriness of antibiotic treatments. Of note, in most cases, the additional administration of anti-inflammatory drugs probably might have resolved hypersensitivity pneumonia or idiopathic $\mathrm{PH}$ (IPH). The most commonly described systemic clinical manifestations were intermittent fever, progressive anorexia and FTT. Inflammatory markers were usually found to be high. Eosinophilia and severe iron deficiency anemia were frequently described at blood count examination. Gastrointestinal manifestations were reported in about half of the patients and included frequent vomiting or diarrhea. Rarely, lymph node hypertrophy with hepatomegaly, splenomegaly and hypertrophied tonsils or adenoids were labeled [7]. Noticeably, lymphonodular hyperplasia in biopsy was found in a child with HS-manifesting hematochezia [18].

Clinically, the disease can be complicated with cardiopulmonary involvement, such as alveolar hypoventilation, massive acute $\mathrm{PH}$, pulmonary hypertension and cor pulmonale, or nephrological ones, such as crescentic glomerulonephritis $[8,13]$. These characteristics contributed drastically to morbidity and emerged in situations of overdue diagnosis and management. In particular, a delayed manifestation of the disease is episodic hemoptysis, which may represent a $\mathrm{PH}$ with repeated episodes of intra-alveolar bleeding, hemosiderin deposition in alveolar macrophages, followed by the development of pulmonary fibrosis and severe anemia [8]. PH may occur as a primary disease of the lung (also called IPH) or secondary to cardiac diseases, bleeding disorders, collagen-vascular diseases or systemic vasculitis. IPH, if not treated, leads to progressive pulmonary fibrosis and may be lethal [21].

\subsection{Pathogenesis and Immunological Implications}

The exact mechanism that triggers HS is not fully understood. Feasibly, the formation of immune complexes is strongly suspected (Gell and Coombs type III reaction) and the cell- 
mediated reaction (Gell and Coombs type IV reaction) may contribute to the development of this challenging disease.

Some cases showed positive skin tests [3,7], high serum total IgE levels [7], high milk-specific IgE antibodies [13,14] or circulating immune complexes [3]. A significant increase of histamine and ECP in BAL several hours after a milk OFC was reported [10]. In one case report, deposits of immunoglobulins, complement, fibrin and milk protein antigens diffusely scattered were described on immunofluorescence studies of lung tissue biopsies [2]. It is probable that a cause concurring to HS is the aspiration of milk, in particular among patients with an uncoordinated swallowing mechanism, tracheal/esophageal anomaly or gastroesophageal reflux. However, in the paper of Boat et al., this condition was ruled out [7]. Concerning the data on delayed immunity, in some cases a delayed skin test response to intradermal test [3,7] or a lymphocyte response [8] was reported. Other authors postulated that milk antigens might trigger an immune complex reaction resulting in multiorgan abnormalities, such as pulmonary, gastrointestinal and renal ones. In fact, pulmonary and gastrointestinal signs and symptoms were frequently associated [11] and granular immuno-deposits have been demonstrated along the glomerular basement membrane in a child with crescentic glomerulonephritis and PH [13].

Most studies, mainly the oldest ones, characteristically found high titers of precipitins (likely immunoglobulins of class $\mathrm{G}$ ) against bovine milk proteins in the patients' sera, by using the Ouchterlony double-immunodiffusion technique $[4,5,7,11]$. However, it is not sufficiently explicable why some children develop precipitating antibodies to ingested protein and other children do not. Moreover, it is not known if these precipitins play a causative role in the disease. Children with precipitins usually have an increased incidence of recurrent respiratory tract diseases, anemia and hepatosplenomegalia. However, precipitating IgG antibodies to milk are not pathognomonic of the disease, since they have been found among around $1 \%$ of healthy children in the absence of clinical manifestations [4], and in $4 \%$ to $6 \%$ of children with chronic disorders, including celiac disease, cystic fibrosis, IgA deficiency, Down's syndrome, Wisckott-Aldrich syndrome and Hurler's syndrome [7]. Additionally, methods used for detecting precipitins are obsolete and reports on them are timeworn. Therefore, the role of these antibodies should be critically considered.

In other more recent reports, high values of specific IgG to milk proteins were found using an immunoenzimatic technique [14]. In one case report [15] CM IgG4 was found to be elevated. Even in these cases, the role of this specific CM IgG is not clear.

\subsection{Pulmonary Modifications and Immunofluorescence Studies}

Chest roentgenograms displayed variable patchy and transient infiltrates, frequently associated with areas of atelectasis, consolidation, reticular opacities, pleural thickening or hilar lymphadenopathy. The lung biopsy obtained in patients who had hemoptysis showed an abnormal accumulation of hemosiderin in the lungs, which resulted in alveolar hemorrhage or $\mathrm{PH}$ [2]. Among patients with anemia and hemoptysis, $\mathrm{PH}$ was verified by the demonstration of iron-laden macrophages by using Prussian Blue staining of bronchial aspirates or morning gastric washes [8].

\subsection{Diagnostic Criteria}

As described in detail above, in order to better stratify the level of evidence for the diagnosis, we are herein proposing the first structured diagnostic approach for the diagnosis of HS to the best of our knowledge. We auspicate that this approach may allow clinicians to stratify patients with a clinical history consistent with the suspicion of HS in probable (criteria A $+\mathrm{B}$ ) or convincing (criteria A $+\mathrm{B}+\mathrm{C}$ ) HS (Figure 1). According to our criteria, only 6 out of 61 patients had a convincing clinical diagnosis of HS, in 25 patients the clinical diagnosis was probable and in the others the HS diagnosis was doubtful (Table 1). Due to the heterogeneity in the methodologies applied for laboratory tests and missing reporting/lack of data, we decided to exclude laboratory parameters from the diagnostic criteria. We suggest in the future that milk-specific IgG tests with 
current diagnostic methods (i.e., immunoenzymatic), in the case of suspected HS, could be studied. Nevertheless, further points remain questionable in our proposed diagnostic approach. First, some cases of occurrence of signs and symptoms during exclusive maternal breastfeeding were reported (e.g., in three out of seven cases in Heiner et al. [3], leading to the question of whether or not minimal quantities of $\mathrm{CM}$ passing in breast milk are capable of inducing a clinical response through an IgG-mediated mechanism in the infant). As a second observation, one case of resolution of signs and symptoms without dietary restriction has been described (one out of seven patients from Heiner et al.). Again, a real, controlled OFC of milk was reported only in 6 cases $[3,7,11]$ and a recurrence of clinical manifestations was reported in a further 6 cases (although without details on exact timing of the symptoms' onset) based only on clinical history $[5-7,13,14,16]$, for a total of 12 cases. Moreover, in some cases $[13,14]$ pharmacological therapy was associated to the CM elimination diet, making it difficult to differentiate the effect of each single treatment. Spontaneous resolution of signs and symptoms also occurred in some cases of HS [1]. Furthermore, some studies did not report on the follow-up and specifically on the outcome of any $\mathrm{CM}$ reintroduction. In conclusion, even if in a few cases a convincing diagnosis can be made using specific criteria, certainty is lacking due to the incomplete clinical and imaging monitoring of the OFC and the lack of control cases.

\subsection{Differential Diagnosis}

In the differential diagnosis (DD) bronchial asthma, chronic aspiration, acute and chronic lower respiratory tract infections, including fungal ones, cystic fibrosis, foreign body, hypersensitivity pneumonitis, bronchopulmonary aspergillosis, secondary $\mathrm{PH}$ and IPH should be considered [11]. Cystic fibrosis essentially can be excluded by a normal chloride level on the sweat test and second line tests if there is a strong suspicion. Infectious causes should be ruled out with tuberculin skin test and available microbial tests, and empiric antibiotic treatments characteristically are inefficacious. Thereafter, a vasculitis or autoimmune disorder can be considered. The characteristic pulmonary hemorrhage attacks cannot be enlightened by modest bronchial asthma. The lack of chronic inhalant exposure and BAL examination can rule out hypersensitivity pneumonitis. Bronchopulmonary aspergillosis is unlikely if skin reactivity to Aspergillus antigens is negative or precipitating serum antibodies to $A$. fumigatus are absent. No hemorrhagic focus nor foreign bodies can be found on bronchoscopy. In particular, DD should consider IPH, that includes the classic triad hemoptysis, radiologic lung infiltrate and iron deficiency anemia, having a more severe course and prognosis [22]. Moreover, IPH occurs in older children, and it is rarely associated with gastrointestinal symptoms [23] (Table 2).

Table 2. Differential diagnosis between Heiner syndrome (HS) and idiopathic pulmonary hemosiderosis (IPH).

\begin{tabular}{ccc}
\hline & HS & IPH \\
\hline Age & infants or young children & older children/adults \\
Hemosiderosis & often & always \\
GI symptoms & often & rarely \\
Precipitins & yes & no \\
Response to diet & yes & no \\
Prognosis & good & variable \\
\hline
\end{tabular}

\subsection{Natural History}

The disease's effects were reversible by stopping CM consumption. In fact, signs and symptoms could last within a time range of 5 to 21 days after the $\mathrm{CM}$ withdrawal. Before CM reintroduction, some children showed spontaneous tolerance to pasteurized or boiled CM [4]. Reoccurrence of clinical manifestations was reported with CM reintroduction $[3,5,6,10,11,14]$. However, it is believed that patients usually definitively outgrow this hypersensitivity, and they can tolerate CM within a few years [11,24,25]. 


\subsection{Treatment}

Clinical improvement with strict elimination of $\mathrm{CM}$ proteins sustains the diagnosis of HS. Infants may be fed by a milk substitute, such as extensively hydrolyzed protein formula, soy-based formula or synthesized free amino acid formula. Improvement of signs and symptoms occurs in few days and X-ray images in weeks. When a confirmatory CM reintroduction was performed [11], respiratory clinical manifestations also occurred in days or weeks. An early exclusion of the triggering food from the diet is crucial, since chronic PH induces pulmonary fibrosis which can be fatal [6]. However, recovery may occur also without exclusion of the culprit food (e.g., 1/7 in Heiner's report [3]).

Even if the most striking criterion of HS is the dramatic response to the exclusion diet, initially, in some cases, appropriate treatment, e.g., bronchodilators, antihistamines, systemic or inhaled steroids and iron, may be needed. A short cycle of oral corticosteroids remains the first-line therapy for acute attacks. In more severe cases of HS, other immunomodulatory treatments may be helpful, such as hydroxychloroquine, azathioprine or cyclophosphamide. On the contrary, antibiotic therapy seems not to be useful.

\section{Conclusions}

Although HS has been described as a hypersensitivity disease due to CM, there are still pros and cons about its real existence (Table 3), and a certainty of diagnosis is lacking. A challenge test was performed in a few cases and always in an open manner. Again, signs and symptoms develop in the hours or days after milk consumption, and they disappear after the elimination diet. Precipitating IgG antibodies are an old-fashioned laboratory test reported in some patients during the disease; precipitins diminish or disappear during the elimination diet. However, they are not pathognomonic nor specific for the disease diagnosis and their pathogenetic role is still unclear. The prognosis is generally good, even if the extent of the exclusion diet necessary to reach a complete recovery is unclear. HS is a very intriguing disease, in some ways still controversial, but it is important to know and suspect this rare syndrome in any infant or young child with unexplained chronic pulmonary clinical manifestations. The diagnosis should be proved by clinical and radiologic improvements after strict $\mathrm{CM}$ avoidance with the recurrence of signs of symptoms and imaging features after a controlled CM reintroduction.

Table 3. The real existence of Heiner syndrome: pros and cons.

\begin{tabular}{|c|c|}
\hline Pros & Cons \\
\hline Multiorgan involvement (in particular lung and GI) & Absence of case-control studies \\
\hline Detection of precipitating antibodies & Precipitating antibodies not pathognomonic \\
\hline Scarce response to non anti-inflammatory drugs & $\begin{array}{l}\text { In most cases the additional administration of anti-inflammatory drugs } \\
\text { probably resolved hypersensitivity pneumonia or } \mathrm{PH}\end{array}$ \\
\hline Clinical improvement after milk removal & The presence of milk in pulmonary infiltrates reported only in one case \\
\hline Symptoms' reoccurrence after milk reintroduction & $\begin{array}{l}\text { Confirmatory challenge not provided in most cases and/or not } \\
\text { adequately performed }\end{array}$ \\
\hline
\end{tabular}

Considering the high clinical impact of the disease and the associated morbidity, more attention should be devoted to it, both in terms of clinical suspicion and research on the underlining patho-mechanisms with the detection of reliable biomarkers. We highlight the need for more stringent diagnostic criteria that combine both clinical manifestations and imaging features. Moreover, a follow-up evaluation with a well-designed CM OFC/regular reintroduction is of paramount importance to better understand this disease in terms of prognosis and duration. In summary, the future establishment of validated diagnostic criteria, the awareness of specific clinical manifestations and specific imaging features and the results of CM OFC will help health professionals in clinical practice to suspect the disease and to refer patients to the appropriate specialists. 
Author Contributions: E.N. conceived the study, coordinated it, helped in drafting the manuscript and critically reviewed it. S.A. and C.M. participated in the study's design, carried out the literature research and wrote the first draft of the manuscript. L.P., F.M., M.G. and S.B. reviewed and provided feedback. All authors have read and agreed to the published version of the manuscript.

Funding: This research received no external funding.

Institutional Review Board Statement: Not applicable.

Informed Consent Statement: Not applicable.

Data Availability Statement: Not applicable.

Acknowledgments: Not applicable.

Conflicts of Interest: The authors declare no conflict of interest.

\section{References}

1. Heiner, D.C.; Sears, J.W.; Kniker, W.T. Multiple precipitins to cow's milk in chronic respiratory disease. A syndrome including poor growth, gastrointestinal symptoms, evidence of allergy, iron deficiency anemia, and pulmonary hemosiderosis. Am. J. Dis. Child 1962, 103, 634-654. [CrossRef] [PubMed]

2. Lee, S.K.; Kniker, W.T.; Cook, C.D.; Heiner, D.C. Cow's milk-induced pulmonary disease in children. Adv. Pediatr. 1978, 25, 39-57.

3. Heiner, D.C.; Sears, J.W. Chronic respiratory disease associated with multiple circulating precipitins to cow's milk. Am. J. Dis. Child. 1960, 100, 500-502.

4. Holland, N.H.; Hong, R.; Davis, N.C.; West, C.D. Significance of precipitating antibodies to milk proteins in the serum of infants and children. J. Pediatr. 1962, 61, 181-195. [CrossRef]

5. Chang, C.H.; Wittig, H.J. Heiner's syndrome. Radiology 1969, 92, 507-508. [CrossRef]

6. Archer, J. Idiopathic Pulmonary Hemosiderosis Treated with a Milk-free Diet. Proc. Roy. Soc. Med. 1971, 64, 1217-1218. [CrossRef] [PubMed]

7. Boat, T.F.; Polmar, S.H.; Whitman, V.; Kleinerman, J.I.; Stern, R.C.; Doeshuk, C.F. Hyperactivity to cow milk in young children with pulmonary hemosiderosis and cor pulmonale secondary to nasopharyngeal obstruction. J. Pediatr. 1975, 87, 23-29. [CrossRef]

8. Stafford, H.A.; Polmar, S.H.; Boat, T.F. Immunologic Studies in Cow's Milk-induced Pulmonary Hemosiderosis. Pediatr. Res. 1977, 11, 898-903. [CrossRef]

9. Fossati, G.; Perri, M.; Careddu, G.; Mirra, N.; Carnelli, V. Pulmonary hemosiderosis induced by cow's milk proteins: A discussion of a clinical case. Pediatr. Medica Chir. Med Surg. Pediatrics 1992, 14, 203-207.

10. Torres, M.J.; Girón, M.D.; Corzo, J.L.; Rodriguez, F.; Moreno, F.; Perez, E.; Blanca, M.; Martinez-Valverde, A. Release of inflammatory mediators after cow's milk intake in a newborn with idiopathic pulmonary hemosiderosis. J. Allergy Clin. Immunol. 1996, 98, 1120-1123. [CrossRef]

11. Moissidis, I.; Chaidaroon, D.; Vichyanond, P.; Bahna, S.L. Milk-induced pulmonary disease in infants (Heiner syndrome). Pediatr Allergy Immunol. 2005, 16, 545-552. [CrossRef]

12. Sigua, J.A.; Zacharisen, M. Heiner syndrome mimicking an immune deficiency. WMJ Off. Publ. State Med Soc. Wis. 2013, 112, 215-217.

13. Yavuz, S.; Karabay-Bayazıt, A.; Yılmaz, M.; Gönlüşen, G.; Anarat, A. Crescentic glomerulonephritis in a child with Heiner syndrome. Turk. J. Pediatr. 2014, 56, 661-664. [PubMed]

14. Mourad, A.A.; Parekh, H.; Bahna, S.L. A 17-month-old patient with severe anemia and respiratory distress. Allergy Asthma Proc. 2015, 36, 506-511. [CrossRef] [PubMed]

15. Alsukhon, J.; Leonov, A.; Elisa, A.; Koon, G. P327 Food-induced pulmonary hemosiderosis. Ann. Allergy Asthma Immunol. 2017, 119, S77. [CrossRef]

16. Ojuawo, A.B.; Ojuawo, O.B.; Aladesanmi, A.O.; Adio, M.O.; Abdulkadir, M.B.; Mokuolu, O.A. Heiner Syndrome: An uncommon cause of failure to thrive. Malawi Med. J. 2019, 31, 227-229. [CrossRef]

17. Koc, A.S.; Sucu, A.; Celik, U. A different clinical presentation of Heiner syndrome: The case of diffuse alveolar hemorrhage causing massive hemoptysis and hematemesis. Respir. Med. Case Rep. 2019, 26, 206-208. [CrossRef]

18. Liu, X.-Y.; Huang, X.-R.; Zhang, J.-W.; Xiao, Y.-M.; Zhang, T. Hematochezia in a Child with Heiner Syndrome. Front. Pediatr. 2020, 7, 551. [CrossRef] [PubMed]

19. Heiner, D.C.; University of California Los Angeles, Torrance, CA, USA. Personal Verbal Communication, 2015.

20. Agata, H.; Kondo, N.; Fukutomi, O.; Takemura, M.; Tashita, H.; Kobayashi, Y.; Shinoda, S.; Nishida, T.; Shinbara, M.; Orii, T. Pulmonary hemosiderosis with hypersensitivity to buckwheat. Ann. Allergy Asthma Immunol. 1997, 78, 233-237. [PubMed]

21. Soergel, K.H.; Sommers, S.C. Idiopathic pulmonary haemosiderosis and related syndromes. Am. J. Med. 1962, 32, 499-511. [CrossRef]

22. Saha, B.K. Idiopathic pulmonary hemosiderosis: A state of the art review. Respir. Med. 2021, 176, 106234. [CrossRef] [PubMed]

23. Taytard, J.; Nathan, N.; De Blic, J.; Fayon, M.; Epaud, R.; Deschildre, A.; Troussier, F.; Lubrano, M.; Chiron, R.; Reix, P.; et al. New insights into pediatric idiopathic pulmonary hemosiderosis: The French RespiRare ${ }^{\circledR}$ cohort. Orphanet J. Rare Dis. $2013,8,161$. [CrossRef] 
24. Lee, E.J.; Heiner, D.C. Allergy to Cow Milk. Pediatrics Rev. 1986, 7, 7. [CrossRef] [PubMed]

25. Williams, S.; Craver, R.D. Cow's milk-induced pulmonary hemosiderosis. J. State Med Soc. Off. Organ State Med Soc. 1989, 141, $19-22$. 